\section{Problem Definition}

Adrenal insufficiency after transsphenoidal resection of pituitary adenoma (PA) can be seen in $1-12 \%$ of cases. In PA, the use of postoperative cortisol measurement and supplementation remains controversial. It is unclear whether postoperative cortisol supplementation has a measurable effect on improving outcomes in patients with pituitary adenoma undergoing endoscopic transsphenoidal surgery (ETS). The objective of the study was to evaluate a postoperative steroid treatment protocol in patients with PA undergoing ETS.

\section{Aims For Improvement}

S: develop a steroid sparing protocol for PA patients undergoing ETS.

M: measure steroid administration and levels, as well as outcomes.

A: achievable because involves small protocol modification.

$\mathrm{R}$ : relevant because this can spare unnecessary medication.

T: test over the period of multiple years.

\section{Intervention}

A retrospective cohort study was performed for patients undergoing ETS from 2005 to 2020 for PA at a single tertiary academic center. Patients were divided into two groups: those managed by a routine postoperative glucocorticoid supplementation protocol (standard protocol) and those that received supplementation based on postoperative cortisol laboratory assessment (sparing protocol). Management was otherwise the same between groups. Both univariate and multivariate analysis was performed.

\section{Measurements/ Results}

Among 535 patients, 21\% ( $\mathrm{n}=111)$ received postoperative steroids, while the remainder $(n=424)$ did not. There were no differences in mean length of stay ( 3 days vs. 3 days, $\mathrm{p}=0.72$ ), sinonasal complaints (27\% vs. $19 \%, \mathrm{p}=0.12$ ), 30-day readmission ( $5 \%$ vs. $5 \%, \mathrm{p}=0.44$ ), and perioperative complications ( $5 \%$ vs. $5 \%$, $\mathrm{p}=0.79$ ) between both the groups. A multivariate model also supported that both groups were comparable in predicting length of stay, 30-day readmission, and complications.

\section{Lessons Learned}

In patients who underwent endoscopic transsphenoidal surgery for pituitary adenoma, there were no differences in outcomes between those on the standard postoperative steroids protocol and those on the postoperative steroid sparing protocol. This suggests that the routine administration of glucocorticoids may not be necessary following surgery for pituitary adenoma.

\begin{tabular}{|c|c|c|c|}
\hline & $\begin{array}{l}\text { Traditional } \\
\text { Steroid Protocol } \\
(\mathrm{N}=111)\end{array}$ & $\begin{array}{l}\text { Steroid Sparing } \\
\text { Protocol }(N=424)\end{array}$ & $\begin{array}{c}P \\
\text { Value }\end{array}$ \\
\hline $\begin{array}{l}\text { Length of Stay, mean } \\
\text { days (sd) }\end{array}$ & $3(2)$ & $3(2)$ & 0.72 \\
\hline $\begin{array}{l}\text { 30-day Readmission, } n \\
(\%)\end{array}$ & $6(5 \%)$ & $21(5 \%)$ & 0.44 \\
\hline Headache, n & 0 & 7 & \\
\hline CSF leak, $n$ & 2 & 4 & \\
\hline Hyponatremia, n & 1 & 5 & \\
\hline Adrenal insufficiency, $n$ & 0 & 3 & \\
\hline Other*, $\mathrm{n}$ & 3 & 2 & \\
\hline $\begin{array}{l}\text { Postoperative sinonasal } \\
\text { complaints, } \mathrm{n}(\%)\end{array}$ & $21(19 \%)$ & $125(27 \%)$ & 0.12 \\
\hline Septal perforation, $\mathrm{n}$ & 2 & 9 & \\
\hline Sinusitis, $\mathrm{n}$ & 16 & 70 & \\
\hline Synechiae, $\mathrm{n}$ & 3 & 37 & \\
\hline $\begin{array}{l}\text { Postoperative SNOT } \\
\text { score, mean (SD) }\end{array}$ & $22(24)$ & $16(16)$ & 0.12 \\
\hline $\begin{array}{l}\text { Perioperative } \\
\text { Complications, n (\%) }\end{array}$ & $6(5 \%)$ & $20(5 \%)$ & 0.79 \\
\hline Epistaxis, $\mathrm{n}$ & 2 & 5 & \\
\hline Meningitis, $\mathrm{n}$ & 0 & 0 & \\
\hline Pulmonary embolism, n & 2 & 3 & \\
\hline Sellar hemorrhage, n & 2 & 2 & \\
\hline Thromboembolism, $\mathrm{n}$ & 0 & 10 & \\
\hline
\end{tabular}

